\title{
DISTRIBUIÇÃO E EXPANSÃO EM UM MODELO NEOESTRUTURALISTA: UMA APLICAÇÃO PARA O BRASIL ${ }^{(*)}$
}

\author{
Henrique Morrone ${ }^{a}$ \\ Adalmir Antônio Marquetti $i^{b}$
}

RESUMO: Este artigo apresenta um modelo neo-estruturalista unisetorial de equilíbrio geral para investigar os efeitos de choques em variáveis econômicas sobre o desempenho da economia brasileira. A matriz de contabilidade social para 2006 foi construída empregando os dados do Sistema de Contas Nacionais (SCN). Três experimentos são analisados: uma desvalorização cambial; uma transferência de renda aos trabalhadores; e a combinação da desvalorização cambial com transferências de renda aos trabalhadores. Os resultados indicam efeitos positivos da política que combina a desvalorização cambial e a transferência de renda governamental na economia brasileira.

PALAVRAS-CHAVE: Modelo neoestruturalista; matriz de contabilidade social.

CLASSIFICAÇÃO JEL: O11; O41; F41.

* Artigo recebido em 22/07/2013 e aprovado em 26/11/2013.

a PhD em Economia pela Universidade de Utah (EUA), professor adjunto da Universidade Federal do Rio Grande do Sul (UFRGS). Contato: hmorrone@hotmail.com.

b PhD em Economia pela New School (EUA) e professor da Pontifícia Universidade Católica do Rio Grande do Sul (PUC/RS). Contato: aam@pucrs.br. 


\title{
DISTRIBUTION AND EXPANSION IN A NEOSTRUCTURALIST MODEL: AN APPLICATION TO BRAZIL
}

\begin{abstract}
This paper presents a neo-estruturalist model that attempts to evaluate the impact of simulation exercises on the economic performance of the Brazilian economy. The data from the National Accounting System is employed to build a Social Accounting Matrix that serves as base for our model. Three experiments are applied: a rise in the investment level, an income transfer to workers, and a policy mix that combines both the preceding simulations (devaluation with redistribution). The results suggest that every experiment impacts positively the Brazilian economy; it validates the neo-structuralist claims about the positive effects of exchange devaluation and redistribution on the level of economic activity.
\end{abstract}

KEYWORDS: Neo-estruturalist model; social accounting matrix. 


\section{INTRODUÇÃO}

O aumento da taxa de crescimento do produto no período 2003-2008 no Brasil reflete a ação conjunta de fatores potencializadores da expansão econômica. A combinação de um cenário internacional positivo, pautado pela elevação dos preços e demanda das commodities (decorrentes da ascenção da China e da Índia, juntamente com especulação nos mercados internacionais), contribuiu para os resultados positivos do período supracitado. No plano doméstico, a expansão do crédito e o Programa de Aceleração do Crescimento (PAC) foram vitais para o aumento da atividade econômica. $\mathrm{O}$ PAC representou a retomada do papel do estado no planejamento econômico e na coordenação dos investimentos públicos e das empresas estatais e privadas. A taxa de investimento apresentou rápida recuperação após a adoção do programa, passando de 15,9\% em 2005 para 19,5\% em 2010. Esses fatores em conjunto foram responsáveis pelo aumento do emprego, com crescimento econômico e inclusão social.

Apesar dos resultados positivos, a economia apresentou uma apreciação cambial ${ }^{1}$ de 2004 a meados de 2011, o que impactou o desempenho da economia nacional. A diferença entre a taxa de juros dos títulos da dívida pública brasileira em relação às taxas internacionais foi fator importante de atração de recursos financeiros e, portanto, de apreciação do real. A taxa de câmbio sobrevalorizada afeta de várias formas a economia. Primeiro, ela aumenta o déficit em conta corrente e a necessidade de atrair poupança externa através da conta de capital, o que resulta no aumento do passivo externo. Segundo, ela reduz a competitividade da indústria, o que acelera o processo de desindustrialização no médio e longo prazo. Contudo, a apreciação cambial facilita a aquisição de máquinas e equipamentos pelo setor industrial, podendo gerar o aumento de produtividade. Ademais, a taxa de câmbio apreciada evita um maior crescimento dos preços, com efeitos positivos sobre o salário real e, portanto, sobre o consumo. Isso pode atuar como um fator de estímulo ao crescimento em economias de grande porte, detentoras de amplo mercado interno. Entretanto, não há consenso na literatura especializada quanto ao efeito líquido da apreciação cambial na atividade produtiva nacional. Estimar o efeito de políticas econômicas na economia nacional é um dos objetivos desse artigo.

Neste artigo, apresentamos um modelo macroeconômico neoestruturalista a fim de avaliar os impactos de políticas macroeconômicas no Brasil. O modelo segue a tradição neoestruturalista, na qual a demanda possui um papel central na explicação do processo expansionário das economias. Os antecessores do modelo são Taylor (1983), Dutt

1 Os termos apreciação (depreciação) e valorização (desvalorização), nesse estudo, são usados de forma intercambiável, independente do regime cambial adotado. 
(1984), e von Arnim (2010). Utilizando a matriz de contabilidade social do Brasil de 2006, o modelo representará o comportamento esquemático do lado real da economia brasileira. Nessa linha, três experimentos são testados: uma desvalorização cambial, um aumento da transferência de renda do governo aos trabalhadores,e uma política que combina desvalorizações cambiais com transferências de renda aos trabalhadores.

O estudo inova ao mensurar os impactos de desvalorizações cambias e políticas redistributivas na economia real. Uma proposta neoestruturalista refere-se aos possíveis efeitos benéficos de desvalorizações acompanhadas de transferências de renda governamentais, que através do aumento das exportações, alavancam a produção nacional. Desse modo, mensurar os efeitos dessas políticas torna-se o foco desse estudo.

O restante do artigo está estruturado da seguinte forma. No próximo item, apresenta-se uma breve revisão teórica. $\mathrm{O}$ item 3 apresenta o modelo. $\mathrm{O}$ item 4 aborda os resultados das simulações, incluindo a análise de sensitividade dos resultados a mudanças nas elasticidades-preço do comércio exterior. $\mathrm{O}$ último item reserva-se as conclusões.

\section{NEOESTRUTURALISMO, DISTRIBUIÇÃO E EXPANSÃO: UMA BREVE REVISÃO}

Os modelos de equilíbrio geral computável (neo)estruturalista (em inglês: Structuralist Computable General Equilibrium - SCGE) foram aplicados inicialmente em economias em desenvolvimento em meados dos anos 1970. Também conhecidos como modelos pós-keynesianos calibrados, estes modelos foram empregados no Brasil no início da década de 1980. Taylor e Lysy (1980) foram os pioneiros, desenvolvendo um modelo a fim de investigar os resultados econômicos de mudanças na distribuição de renda do país. Posteriormente, o foco das pesquisas mudou para questões relacionadas com a restrição externa e as políticas de estabilização.

Esses modelos foram influenciados pelas contribuições de Lewis (1954), Prebisch (1959), Kalecki (1971)e Leontief (1983). O conceito de setores que se comportam de forma diferenciada e da economia dual empregado por estruturalistas foi primeiramente desenvolvido pelos pré-clássicos e Lewis, respectivamente. O tratamento da restrição externa foi fortemente influenciado pelos estudos de Prebisch. Modelos estruturalistas norte-sul, empregados para descrever a interrelação entre países da periferia e do centro, geralmente levam em consideração as condições de termos de comércio desfavoráveis. Por fim, Kalecki e Leontief também influenciaram o processo de modelagem estruturalista. A matriz de insumo-produto e a teoria de preços do mark-up foram incorporadas na maioria dos modelos de simulação estruturalista.

Para neoestruturalistas, desenvolvimento econômico resulta de uma profunda mudança estrutural na economia em direção a atividades com economias de escala 
dinâmicas ${ }^{2}$. Estruturalismo considera a estrutura da economia como sendo um fator central na explicação do efeito de choques econômicos (Taylor, 1990). Nesse sentido, fatores como, por exemplo, a distribuição de renda, as relações intersetoriais e as relações de classes são importantes elementos na formulação do modelo estruturalista. $\mathrm{O}$ estudo das instituições de cada economia, bem como aspectos históricos e de política econômica são cruciais no processo de formulação do modelo. Ademais, os modelos neoestruturalistas compartilham os seguintes pontos:

1. Conflito de classes existe e pode desencadear processos inflacionários.

2. Distribuição é considerada exógena no curto e médio prazo.

3. Demanda explica o crescimento (fechamento Keynesiano), refutando a Lei de Say. Nesse sentido, o equilíbrio macroeconômico não está atrelado ao pleno emprego.

4. Investimento depende da distribuição de renda e do acelerador.

5. Modelo deve apresentar consistência contábil com as contas nacionais e as matrizes de insumo-produto. Exercícios de estática comparativa são empregados a fim de mensurar o impacto de choques econômicos na economia.

Os modelos neoestruturalistas e neokaleckianos foram aplicados em uma série de economias a fim de investigar as especificidades do processo de crescimento, dentre elas a relação existente entre redistribuição de renda e crescimento. Inicialmente, Dutt (1984) construiu um modelo que descrevia a relação entre crescimento e distribuição de renda para a Índia. Seus resultados (uma relação positiva entre crescimento e distribuição) contrariaram a literatura do desenvolvimento econômico convencional baseada em variações da equação de Cambridge. $\mathrm{O}$ argumento padrão é o que segue: quanto maior o crescimento do produto, maiores serão os requerimentos para acumulação, os quais, consequentemente, dependerão de poupança para sustentar o crescimento. Desse modo, redistribuição de renda em favor dos capitalistas é uma necessidade. Esses modelos assumem implicitamente que a economia opera em pleno emprego. Quando assumimos a existência de excesso de capacidade, os resultados encontrados por Dutt para a Índia tornam-se uma possibilidade. Ele verificou que um nível insuficiente de demanda seria central para explicar a estagnação da economia indiana durante as décadas de 1960 e 1970. Nesse contexto, políticas redistributivas de renda engendrariam crescimento com igualdade. Taylor (1985) encontrou resultados similares com seu modelo wage-led (estagnacionista). Assim, economias poderiam

\footnotetext{
2 Economias de escala dinâmicas são geradas pelo progresso técnico, learning by doing, economias externas e divisão do trabalho. O processo de aprendizado pode melhorar a capacidade inovativa.
} 
apresentar regimes de crescimento da demanda diferenciados: algumas economias responderiam ao crescimento dos salários, por isso wage-led; em outras os lucros seriam o motor do crescimento (profit-led).

Entretanto, existeuma importante qualificaçãoao modelo neokaleckiano. Blecker (1989) argumenta que economias abertas ao exterior tenderiam a apresentar uma relação inversa entre salários e lucros. Nesse contexto, as firmas levariam em consideração a competição externa antes de determinar seus mark-ups. Mark-ups seriam estrategicamente fixados a fim de obter competitividade internacional e penetrar em mercados externos. Caso este argumento seja válido, uma redistribuição de renda em direção aos trabalhadores causaria a redução dos lucros uma vez que as firmas não conseguiriam repassar o aumento dos custos para os consumidores. Ou seja, qualquer crescimento nos preços finais será traduzido em uma redução no market-share doméstico e internacional.

Desse modo, uma desvalorização cambial pode causar tanto uma contração quanto uma expansão do nível de atividade econômica. Uma desvalorização cambial seria contracionária para economias em desenvolvimento que apresentem importação de bens de capital preço inelásticas. Outro possível fator que poderia causar a contração do produto é que uma depreciação cambial reduz o salário real, consequentemente, diminuindo o consumo. Krugman e Taylor (1978) apresentam uma discussão detalhada desses fatores.

Taylor (1983) aplicou um modelo SCGE para a Índia, detectando que depreciações cambiais seriam contracionárias. No contexto indiano, a depreciação eleva o peso das importações, as quais geram o aumento dos custos intermediários do setor industrial. Este crescimento nos custos causa a elevação dos preços finais, reduzindo os salários reais e a demanda agregada. Uma vez que os setor industrial atinge o equilíbrio via ajuste nas quantidades (o setor apresenta excesso de capacidade), o produto industrial declinará substancialmente.

Em linhas gerais, uma apreciação cambial (aumentadora dos custos internos relativos ao exterior) geraria a queda dos lucros com repercussões negativas no investimento. Esse resultado negativo poderia ser neutralizado caso a economia siga um regime wage-led. Nesse caso, o crescimento do salário real alavancaria o consumo, impactando positivamente o investimento e o nível de atividade econômica.

Já uma desvalorização cambial (geradora da redução dos custos domésticos em relação ao exterior) engendraria a redistribuição de renda em favor dos capitalistas, impactando positivamente o investimento e o nível de atividade caso o regime de crescimento seja liderado pelos lucros (ou profit-led). Logo, desvalorizações cambiais seriam expansionárias para regimes profit-led, enquanto desvalorizações produziriam um efeito contracionário caso o regime de crescimento seja wage-led.

Assim, políticas de câmbio competitivo devem ser efetuadas com cautela, tendo em vista queo aumento nos custos de produção e as reduções salariais podem gerar 
resultados contracionários caso as exportações não sejam fortemente elásticas à desvalorização cambial. A princípio, economias com um mercado doméstico amplo e com um nível relativamente modesto de abertura comercial tendem a ser fracamente wage-led. Contudo, regimes profit e wage-led ${ }^{3}$ são características de curto e médio prazo das economias, devendo o exame do regime de crescimento estar necessariamente atrelado ao padrão das transferências de renda interinstitucionais existentes na economia em questão.

\section{MODELO}

O presente item apresenta o modelo neo-estruturalista de curto prazo. Os antecessores do modelo incluem os trabalhos de Taylor (1983), Dutt (1984) e von Arnim (2010). O modelo representa uma economia aberta, unisetorial, com excedente de trabalho e duas classes sociais, capitalistas e trabalhadores. De acordo com a tradição Clássica, os capitalistas não consomem; em contraste, os trabalhadores consomem a maior parte da renda (Foley, 2008). A economia produz apenas um bem, que pode ser consumido, investido e exportado. Um fechamento keynesiano é empregado, onde a demanda será a variável indutora da expansão. Ou seja, adota-se a causalidade do investimento para a poupança, e não vice-versa.

Duas hipóteses do modelo se referem à formação dos preços e ao mercado de trabalho. Assume-se que existem desemprego e excesso de capacidade produtiva na economia. Distúrbios na demanda serão acomodados via mudanças nas quantidades produzidas e preços. Com efeito, adota-se um mark-up fixo para descrever o comportamento dos preços da economia. O modelo descreve o ajustamento da economia a choques exógenos no curto e médio prazo, assumindo o estoque de capital constante.

Emprega-se uma função investimento endógena Kaleckiana, respondendo positivamente (negativamente) ao nível de lucro (salários) da economia. Especificamente, a função investimento tem três componentes: o animal spirits (investimento autônomo); a parcela do lucro; e o acelerador. Assume-se que lucros esperados sejam iguais a lucros correntes por simplicidade.

$$
I_{t}=\chi_{0}\left(\pi_{t} Y_{t}\right)^{\chi^{1}}\left(Y_{t}\right)^{\chi^{2}}
$$

3 De um modo geral, esses modelos apresentam instabilidade dinâmica estrutural no longo prazo (Taylor, 1983; 1991). 
Sendo $\chi_{0}$ a medida dos espíritos animais ao estilo de Keynes, $\pi_{t}$ é a parcela do lucro no produto, e $Y_{t}$ é o valor adicionado. A função investimento em nível segue Taylor (1991). Para um modelo teórico de longo prazo que incorpora uma função de acumulação endógena ver Oreiro e Araújo (2013).

No curto prazo, a produtividade do trabalho responde positivamente ao nível de atividade econômica. Isso deve-se ao fato das firmas reterem trabalho qualificado na recessão, derivado dos elevados custos com contratação e treinamento na fase expansionária, explicando o aumento da produtividade durante a expansão. Isto ocorre porque o trabalho antes subutilizado passa a ser utilizado intensamente durante a expansão. A equação a seguir representa esse processo.

$$
\xi=\delta_{0} Y_{t}^{\delta_{1}}\left(\frac{w_{t}}{Z_{t}}\right)^{\delta_{2}}
$$

Onde $\xi$ é a produtividade do trabalho, $Y_{t}$ é o valor adicionado, $\delta_{1}$ é a elasticidade produtividade-produção (também conhecida como elasticidade de Verdoorn), $Z_{t}$ é o preço do valor adicionado, e $w_{t}$ é o salário nominal. O último termo da equação, $\frac{w_{t}}{Z_{t}}$, expressa uma relação positiva entre custos e intensidade do trabalho. Capitalistas exigirão que os empregados trabalhem em um ritmo mais acelerado em resposta ao aumento do salário real. A seguir são apresentadas as equações de quantidades e preços do modelo. (Ver Apêndice para uma lista completa das equações e glossário das variáveis.)

\subsection{PRODUTO E EMPREGO}

A produção real da economia, $X_{t}$, determinada pelos componentes da demanda total. Ou seja, a oferta é uma função dos insumos utilizados na produção, do consumo, do investimento, das exportações e dos gastos do governo. O balanço setorial estabelece que a oferta será igual a demanda, sendo o equilíbrio alcançado através de mudanças nas quantidades e preços.

$$
a_{t t} X_{t}+C_{t}+I_{t}+E_{t}+G_{t}-X_{t}=0
$$

Onde $a_{t t}$ representa o coeficiente de Leontief; $X_{t}$ a produção; $C_{t}$, o consumo; $I_{t^{t}}$ o investimento; $E_{t^{\prime}}$ as exportações; e $G_{t^{\prime}}$ o gasto público.

A função consumo, $C_{t}$ segue a tradição Keynesiana, sendo uma função positiva da renda dos trabalhadores e da razão entre o preço do valor adicionado $\left(Z_{t}\right)$ e do preço de produção $\left(P_{t}\right)$. Em termos formais a função toma a seguinte forma: 


$$
C_{t}=\frac{\left(1-t_{w}-s_{w}\right)\left(Z_{t} w_{t} L_{t}+C a p W+R m W+S u b W\right)}{P_{t}}
$$

Na qual $t_{w}, s_{w}, L_{t}, C a p W, R m W$, e $S u b W$ são, respectivamente, a taxa de impostos sobre os trabalhadores, a taxa de poupança, o número de empregados, as transferências dos capitalistas para o trabalho, as transferências do resto do mundo para os trabalhadores e, por fim, as transferências do governo aos trabalhadores.

$O$ produto, $Y_{t}$, é proporcional a quantidade ofertada, $X_{t}$. Nesse sentido, o valor adicionado é determinado por $Y_{t}=v X_{t}$. Caso não haja alterações da propensão marginal para importar e taxa de câmbio, o valor da parcela do valor adicionado na oferta permanece constante. Podemos expressar essa parcela na equação disposta a seguir:

$$
v=Y_{t} / X_{t}=1-a_{t t}-f e-t t^{X}
$$

Onde $f$ é a propensão marginal para importar; $f=M_{t} / X_{t} ; e$, a taxa de câmbio nominal; e $t t^{X}$, o imposto sobre a produção.

As funções de comércio exterior são equações comportamentais, sendo as exportações afetadas pela demanda externa e taxa de câmbio. Importações respondem a taxa de câmbio e demanda. As equações seguem as especificações adotadas pela literatura neoestruturalista para países em desenvolvimento, com destaque para os trabalhos de Taylor (1990) e von Arnim e Rada (2011).

$$
\begin{aligned}
& E_{t}=\phi \rho^{\phi^{1}} X_{f} \\
& M_{t}=\varphi \rho^{\varphi^{1}} X_{t}
\end{aligned}
$$

Sendo que $\rho$ representa a taxa de câmbio real, $\rho=\left(e P^{*}\right) / P_{t}$, na qual $P^{*}$ é o preço externo e $P_{t}$ é o preço do bem doméstico. $X_{f}$ é a demanda externa, e $X_{t}$ é a produção doméstica. Os parâmetros $\phi$ e $\varphi$ representam, respectivamente, as elasticidades preço das exportações e importações. Por fim, os gastos públicos são considerados exógenos.

\subsection{PREÇOS E DISTRIBUIÇÃO}

O modelo possui três preços $\left(P_{t}, Z_{t}, e\right)$ e três variáveis distributivas $\left(w_{t}, r_{t}, \pi_{t}\right)$ : o preço de oferta $\left(P_{t}\right)$; o preço do valor adicionado $\left(Z_{t}\right)$; a taxa de câmbio nominal $(e)$; o salário nominal $\left(w_{t}\right)$; a taxa de lucro $\left(r_{t}\right)$; e a parcela dos lucros no produto $\left(\pi_{t}\right)$. 
Iniciando pelo preço de produção, $P_{t}$, o mesmo é expresso por uma relação contábil e determinado pelos custos de produção. Isto é, o preço é uma média ponderada dos custos de produção localizados na primeira coluna da Matriz de Contabilidade Social (MCS).

$$
P_{t}=\frac{v Z_{t}+f e}{1-a_{t t}}
$$

A equação (8) define o preço do valor adicionado, $Z_{t}$, como uma equação comportamental que assume um mark-up fixo sobre os custos. O preço do valor adicionado, $Z_{t}$, é uma função da relação lucro produto $\left(\pi_{t}\right)$, dos salários $\left(w_{t}\right)$ e da produtividade $(\xi)$. A razão entre salários e produtividade expressa os custos salariais unitários de produção.

$$
Z_{t}=\frac{1}{\left(1-\pi_{t}\right)} w_{t} / \xi
$$

Sendo $w_{t} \mathrm{e} \xi$ os salários nominais e a produtividade do trabalho, respectivamente. Em resumo, o preço do valor adicionado, $Z_{t}$, responde a mudanças na distribuição funcional da renda e custos salariais; e o preço de oferta, $P_{t}$, responde a mudanças nos custos.

No que se refere ao mercado de trabalho, assume-se a existência de desemprego na economia. O número de empregados é uma função positiva da razão entre o valor adicionado e a produtividade, $L_{t}=\frac{Y_{t}}{\xi}$. O crescimento econômico implica a contratação de trabalhadores no processo produtivo, gerando mais emprego na economia. De acordo com a especificação neokaleckiana de von Arnim (2010), o salário nominal, equação disposta a seguir, é uma função positiva da taxa de emprego.

$$
w_{t}=w_{0}\left(\frac{L_{t}}{L}\right)^{w_{1}} \xi^{w_{2}}
$$

Onde $L_{t}$ é a quantidade de trabalhadores empregados, e $L$ é a força de trabalho.

À medida que o número de trabalhadores aumenta durante a fase expansionário do ciclo, aumenta o poder de barganha por maiores salários. Além disso, o último termo da equação salarial, $\xi$, revela que os trabalhadores exigem maiores salários em resposta aos aumentos da produtividade a fim de participar do progresso. $\mathrm{O}$ aumento do emprego, decorrente do aumento da produção, engendrará conflito entre as duas classes sociais sobre a repartição do excedente. Esse conflito pode levar a pressões inflacionárias, as quais poderão limitar o crescimento. 
A taxa de lucro é uma função positiva do produto e do preço do valor adicionado. O profit share é exógeno no curto prazo.

$$
r_{t}=\pi_{t} \frac{Z_{t} Y_{t}}{P_{t} K_{t}}
$$

Onde $\pi$ é a relação lucro-produto, e $K_{t}$ é o estoque de capital. Assume-se que o estoque de capital é constante no curto e médio prazo.

Por fim, o equilíbrio macroeconômico é dado pela igualdade entre poupança e investimento. Poupança é ofertada pelos capitalistas, pelo setor externo, pelo governo, e pelos trabalhadores. O fechamento do modelo incorpora elementos keynesianos, pois a demanda assume um papel central no processo de crescimento.

\section{DADOS, CALIBRAGEM E RESULTADOS}

Neste item são apresentados os dados, a calibragem e os resultados. Os três experimentos são analisados: uma desvalorização cambial de $10 \%$; o crescimento de $10 \%$ das transferências de renda do governo aos trabalhadores; e um choque que combina desvalorização cambial de $10 \%$ com políticas de transferência de renda. Contudo, a apresentação dos dados e do processo de calibragem torna-se necessária antes de analisarmos os resultados das simulações.

\subsection{DADOS E CALIBRAGEM}

A MCS utilizada no presente trabalho descreve o fluxo da renda na economia brasileira em 2006. A matriz abrange o total das transações da economia. A MCS foi construída a partir do Sistema de Contas Nacionais (SCN-IBGE, 2011) e utilizaram-se os dados da Tabela de Recursos e Usos (TRU) e das Contas Econômicas Integradas (CEI) para a construção da Matriz de Insumo-Produto e da MCS. A metodologia para a construção da MCS é baseada nos trabalhos de Guilhoto e Sesso (2005), Grijó e Berni (2006) e Morrone (2012).

Na coluna (a) da MCS, abaixo das transações intermediárias, temos os custos de produção (salários, lucros, impostos do governo e importações). A coluna (b) mostra o consumo, os impostos e a poupança dos trabalhadores. A coluna (c) pode ser lida da mesma forma, exibindo quanto os capitalistas transferem para outros segmentos da sociedade, pagam de impostos e poupam. Da coluna d à f encontram-se os valores referentes ao governo, exportações e investimento, respectivamente. 
Tabela 1 - Matriz de contabilidade social do Brasil para o ano de 2006 (em bilhões de reais)

\begin{tabular}{|c|c|c|c|c|c|c|c|}
\hline \multirow{3}{*}{$\begin{array}{c}\text { MSC } 2006 \\
\text { (bilhões de reais) }\end{array}$} & \multirow{2}{*}{$\begin{array}{l}\text { Custos } \\
\text { Setor }\end{array}$} & \multirow[b]{2}{*}{ Consumo } & \multicolumn{3}{|c|}{ Usos da renda } & \multirow[b]{2}{*}{ Investimento } & \multirow[b]{2}{*}{ Total } \\
\hline & & & Capitalistas & Governo & Exportações & & \\
\hline & (a) & (b) & (c) & (d) & (e) & (f) & (g) \\
\hline (1) Setor & $1.636,6$ & $1.321,9$ & & 473,6 & 314,6 & 371,4 & $4.118,1$ \\
\hline (2) Trabalho & $1.097,1$ & & 367,7 & 160,6 & 0,4 & & $1.625,8$ \\
\hline (3) Empresas (lucros) & 910,2 & & & & & & 910,2 \\
\hline (4) Governo & 202,6 & 189,1 & 138,7 & & 25,8 & 25,6 & 581,8 \\
\hline (5) Importações & 271,7 & & 35,6 & 14,0 & & & 321,3 \\
\hline (6) Poupança & & 114,8 & 368,1 & $-66,3$ & $-19,6$ & $-397,0$ & 0,0 \\
\hline (7) Total & $4.118,1$ & $1.625,8$ & 910,2 & 581,8 & 321,3 & 0,0 & \\
\hline
\end{tabular}

Fonte: Elaboração própria.

A estimativa dos parâmetros do modelo foram obtidas diretamente dos dados das Contas Nacionais do IBGE, de estudos econométricos, ou calculados residualmente. Como referido acima, as variáveis endógenas foram obtidas para o ano base de 2006 diretamente dos dados da Matriz de Contabilidade Social.

No topo da Tabela 2 observam-se os valores para os parâmetros e variáveis no ano de 2006. A taxa de lucro foi escolhida ad hoc, utilizando-se o valor de 0,25 (ou 25\%). Valor próximo ao utilizado nos estudos empíricos de Taylor (1983), Taylor (1990) e Arnim e Rada (2011) para países em desenvolvimento. A relação lucro-produto $(0,45)$, $\pi_{t}$, foi estimada diretamente da Tabela 1, procedendo-se a divisão do lucro total pelo valor adicionado. Esses valores referentes à parcela lucro-produto e a taxa de lucro foram utilizados para a estimação do estoque de capital da economia, sendo o mesmo calculado como um resíduo. Ou seja, estimou-se o estoque de capital da economia como sendo equivalente a razão entre os lucros totais e a taxa de lucro por simplicidade.

A parcela do produto na oferta total $(v)$, equivalente a 0,48 no ano base, foi obtida como o resultado da divisão do valor adicionado $\left(Y_{t}\right)$ pela produção $\left(X_{t}\right)$. Nessa linha, a variável produtividade do trabalho, $\xi$, também foi obtida diretamente da Matriz de Contabilidade Social (MCS), sendo estimada pela razão entre o valor adicionado $\left(Y_{t}\right)$ e o pessoal empregado $\left(L_{t}\right)$.

$\mathrm{Na}$ modelagem assumiu-se que os trabalhadores consomem uma parcela constante de sua renda, ensejando com isso uma taxa de poupança positiva e igual a $7 \%$. A taxa de poupança capitalista, $s_{\pi}$, foi obtida diretamente dos dados das Contas Nacionais, sendo determinada pela subtração do lucro pelos impostos e outras remessas, sendo o resultado posteriormente dividido pela renda dos capitalistas. Nesse sentido, o modelo segue parcialmente a tradição clássica ao considerar o consumo dos capitalistas irrelevante (Foley, 2008). 
Tabela 2 - Principais parâmetros

\begin{tabular}{c|c}
\hline Variáveis e parâmetros & Brasil \\
\hline$r_{t}$ & 0,25 \\
\hline$\pi_{t}$ & 0,45 \\
\hline$v$ & 0,48 \\
\hline$S w$ & 0,07 \\
\hline$t t^{w}$ & 0,05 \\
\hline$f$ & 0,06 \\
\hline$a_{t t}$ & 0,39 \\
\hline$w_{1}$ & 1,10 \\
\hline$w_{2}$ & 0,30 \\
\hline$\delta_{1}$ & 0,35 \\
\hline$\delta_{2}$ & 0,20 \\
\hline
\end{tabular}

Fonte: Elaboração própria.

A taxa de impostos $\left(t t^{\mathrm{X}}\right)$ foi estimada como sendo a razão entre os impostos produtivos $(T)$ sobre a produção $\left(X_{t}\right)$, sendo igual a 0,05 . De forma análoga, a propensão a importar $(f)$, foi calculada como a razão entre as importações $\left(M_{t}\right)$ e a produção $\left(X_{t}\right)$, sendo igual a 0,06 .

Na base da Tabela 2, constam outros importantes parâmetros do modelo. O coeficiente técnico de Leontief $\left(a_{t t}\right)$ foi estimado como a razão entre o consumo intermediário sobre a produção $\left(X_{t}\right)$, assumindo o valor de 0,39 no ano base.

Para o mercado de trabalho, considerou-se para o ano base de 2006 uma taxa de emprego, $(L t / L)$, de 0,85 (ou 85\%) da força de trabalho na economia. Os parâmetros $w_{1}$ e $w_{2}$ apresentam os valores de 1,1 e 0,3 , respectivamente. Os mesmos foram retirados ad hoc dos estudos de Taylor (1990) e Arnim (2010) para países em desenvolvimento.

Ademais, a calibragem dos parâmetros de duas equações merece destaque. Primerio, adotou-se na equação 2 o valor de 0,35 para a elasticidade produtividade-produção, $\delta_{1}$, tendo em vista que a literatura internacional define que o parâmetro para economias em desenvolvimento estaria próximo de 0,4 (McCombie, 1983; Thirlwall, 1983). Nesse sentido, fomos conservadores na calibragem deste parâmetro. Empregou-se o valor 0,2 para o parâmetro $\delta_{2}$. O parâmetro $\delta_{0}$, medida de outros fatores que afetam a produtividade, foi calculado como um resíduo; ou seja, de posse dos valores para a produtividade, preços, salários e elasticidade de Verdoorn para 2006, nós resolvemos a equação para $\delta_{0}$.

A equação do investimento, equação (1), segue a função proposta por Taylor (1991), tendo como componentes o acelerador, a parcela lucro-produto, e uma parcela autônoma (ou animal spirits). Os parâmetros dessa equação foram escolhidos ad hoc, empregando- 
-se os valores utilizados em Naastepad (2006) e Rada (2012). Nesse sentido, o parâmetro do acelerador $\left(\chi_{2}\right)$ foi considerado com o valor de 0,9 , pois geralmente o acelerador é próximo de 1 . O parâmetro $\chi_{1}$, medida do efeito do nível dos lucros no investimento, assumiu o valor de 0,4. Segundo Taylor (1983), o acelerador é o principal componente explicativo do nível de investimento, sendo superior ao efeito do lucro no investimento para países em desenvolvimento. Por fim, o investimento autônomo (ou animal spirits) foi calculado residualmente; ou seja, de posse dos outros parâmetros e dos valores para o ano base (2006), resolveu-se a equação (1) para obter o animal spirits $\left(\chi_{0}\right)$.

\subsection{RESULTADOS}

Neste subitem três experimentos são analisados: uma desvalorização cambial de $10 \%$, o cerscimento de $10 \%$ das transferências de renda do governo aos trabalhadores, e um choque que combina desvalorização cambial de $10 \%$ com políticas de transferência de renda.

Esses experimentos foram escolhidos devido aos seus possíveis efeitos positivos no nível da atividade econômica. Por exemplo, mensurar o impacto de choques cambias e distributivos é crucial para guiar os rumos da política econômica nacional.

Uma dificuldade encontrada no presente estudo foi obter estimativas confiáveis para as elasticidades-preço do comércio exterior. Estudos econométricos apontam resultados diferenciados, o que prejudica a confiabilidade das estimações. (Para uma pequena amostra de estudos, ver Holanda (1999), Carvalho (2005) e Santos et al. (2009).)

Para contornar essa limitação, empregaram-se duas calibragens (ou cenários). Na Tabela 2, a calibragem ${ }^{4}(1)$ representa um cenário onde as elasticidades-preço do comércio exterior são zero $(\phi, \varphi,=0)$. Em contraste, a calibragem (2) representa uma economia que possui elasticidades-preço elevadas $(\phi, \varphi=0,7)$. A tabela revela os resultados dos três experimentos no que tange a taxa de crescimento do valor adicionado, a taxa de inflação, ao balanço do setor privado, do setor público e ao resultado da balança comercial. Adicionalmente, apresentam-se as taxas de crescimento da produtividade, dos salários nominais, do consumo e do emprego. As estatísticas estão dispostas em pontos percentuais. Os valores referentes aos parâmetros do modelo encontram-se na base da tabela. Todas as simulações foram realizadas no software Mathematica versão 8.0.

\footnotetext{
4 Ao longo do artigo o termo calibragem e cenário serão empregados de forma intercambiável, expressando os resultados do modelo com elasticidades específicas de comércio exterior.
} 
Por fim, testes de sensitividade foram empregados para verificar a relação entre alterações nas elasticidades-preço de comércio exterior e os resultados dos choques de políticas redistributivas.

\subsubsection{CHOQUE DE PREÇOS E DE DEMANDA: DESVALORIZAÇÃO CAMBIAL E TRANSFERÊNCIAS DE RENDA}

A primeira coluna da Tabela 3 exibe os resultados de uma depreciação de $10 \%$ da taxa de câmbio. O efeito do choque cambial no produto depende da condição de Marshal-Lerner, das estatísticas do ano base, e da flexibilidade dos preços ${ }^{5}$.

Conforme o cenário (1), o qual trata comércio exterior como uma proporção fixa do produto, depreciação é contracionária. Após o choque, o PIB real decresce 2,78\%, e um processo deflacionário emerge. $\mathrm{O}$ resultado privado e o balanço do governo também deterioram. Seguindo a redução da renda, o resultado externo deteriora 1,22 pontos percentuais do PIB.

Esses resultados ocorrem devido a contração do saldo externo, causando a queda do emprego, a redução dos salários, e a diminuição do consumo e da produtividade. Em um contexto marcado por importações preço-inelásticas, a depreciação aumenta o peso (custo) das importações. Formalmente, isto pode ser notado pela parcela do valor adicionado na oferta total, $v$, ser uma função negativa da taxa de câmbio. A redução da demanda e dos preços, portanto, condicionam os resultados negativos desse experimento, provocando sucessivas pressões contracionárias.

Com a incorporação das elasticidades da cenário (2), constata-se uma mudança de sinal dos resultados. No nível macro, o PIB cresce $0,89 \%$; os preços crescem $0,27 \%$. Há um impacto positivo no saldo do setor privado, $\left(\frac{S-I}{P I B}\right)$, visto que o crescimento do produto é acompanhado pelo aumento da poupança. O resultado do governo em relação ao PIB melhora 0,31 pontos percentuais, decorrente da expansão da arrecadação. Nesse contexto, o balanço externo apresenta melhora, alavancado pelas exportações.

Os efeitos do choque externo podem ser sintetizados como segue. A depreciação cambial estimula o crescimento das exportações, gerando um saldo externo positivo. Esta melhora do saldo, por sua vez, contribui para o aumento do emprego e dos salários, estimulando o consumo em $0,54 \%$. Com o acréscimo na demanda (e produção), ocorre

\footnotetext{
O crescimento da demanda provoca uma pressão nos preços, impactando a taxa de câmbio real e limitando o efeito expansionário do câmbio na atividade produtiva (Von Arnim e Rada, 2011).
} 
o crescimento da produtividade do trabalho: via relação de Okun. O crescimento da demanda e do lucro também impactam positivamente o investimento, ocasionando o aumento do nível da atividade econômica. Logo, esse processo de crescimento das exportações, do emprego, e dos salários fomenta o aumento da atividade econômica.

Apesar dos resultados positivos da calibragem (2), os efeitos da desvalorização cambial na expansão do PIB são limitados, não sendo nenhuma panacéia. A eficácia de uma política de câmbio competitivo dependerá dos valores da elasticidade-preço do comércio exterior. Parece haver um limite para essa política, devendo ser executada com cautela.

Além disso, o sucesso de uma política de câmbio competitivo será influenciado pelo contexto internacional. Quanto maior o número de países perseguindo uma estratégia de desenvolvimento voltado para fora, menor será a probabilidade de essa política ter êxito. Uma economia global onde todos os países mantêm suas taxa cambiais competitivas para promover exportações apresenta um elevado grau de instabilidade (Palley, 2012).

Tabela 3 - Resultado das simulações (em \%)

\begin{tabular}{|c|c|c|c|c|c|c|}
\hline & \multirow{2}{*}{\multicolumn{2}{|c|}{\begin{tabular}{|c} 
Desvalorização Cambial \\
Desvalorização \\
do câmbio (aumento) \\
$(10 \%)$
\end{tabular}}} & \multirow{2}{*}{\multicolumn{2}{|c|}{$\begin{array}{c}\text { Transferência } \\
\text { Transferência de renda } \\
\text { para Trabalhadores } \\
(10 \%) \\
\end{array}$}} & \multirow{2}{*}{\multicolumn{2}{|c|}{$\begin{array}{c}\text { Política Alternativa } \\
\text { Desvalorização }(10 \%) \\
\text { com redistribuição } \\
(10 \%) \\
\end{array}$}} \\
\hline & & & & & & \\
\hline & 1 & 2 & 1 & 2 & 1 & 2 \\
\hline \multicolumn{7}{|l|}{ Estatísticas Macroeconômicas } \\
\hline Taxa de crescimento do PIB & $-2,779$ & 0,897 & 1,094 & 0,990 & $-1,710$ & 1,876 \\
\hline Inflação & $-0,877$ & 0,279 & 0,341 & 0,308 & $-0,538$ & 0,582 \\
\hline Taxa de câmbio real & 10,973 & 9,694 & $-0,339$ & $-0,307$ & 10,595 & 9,363 \\
\hline Balanço privado ( $\Delta$ em pts $\%$ do PIB) & $-0,486$ & 0,149 & 0,236 & 0,219 & $-0,237$ & 0,362 \\
\hline Balanço do Governo (? em pts \% do PIB) & $-0,738$ & 0,314 & $-0,366$ & $-0,396$ & $-1,118$ & $-0,082$ \\
\hline Balanço externo ( $\Delta$ em pts \% do PIB) & $-1,225$ & 0,463 & $-0,130$ & $-0,176$ & $-1,355$ & 0,280 \\
\hline \multicolumn{7}{|l|}{ Taxas de Crescimento } \\
\hline Produtividade do trabalho & $-1,225$ & 0,391 & 0,477 & 0,432 & $-0,752$ & 0,816 \\
\hline Consumo & $-1,701$ & 0,549 & 1,653 & 1,590 & $-0,056$ & 2,132 \\
\hline Emprego & $-1,573$ & 0,503 & 0,614 & 0,556 & $-0,966$ & 1,051 \\
\hline \multicolumn{7}{|l|}{ Parâmetros } \\
\hline Elasticidade preço do comércio exterior & 0 & 0,7 & & & & \\
\hline Elasticidade de Kaldor-Verdoorn & 0,35 & 0,35 & & & & \\
\hline
\end{tabular}

Notas: Calibragem (1) representa um cenário com produtividade endógena $(0,35)$ e elasticidades-preço de comércio exterior zerada. Calibragem (2) exibe uma situação onde tanto elasticidades-preço como produtividade estão ativadas. Todas as simulações foram realizadas no software Mathematica 8.0.

Fonte: Elaboração própria com base em dados do SCN-IBGE (2011).

Quais são os resultados macroeconômicos de uma transferência governamental de renda de $10 \%$ aos trabalhadores? Para a calibragem (1), o PIB real cresce aproximadamente $1,09 \%$, e a inflação é de apenas $0,34 \%$. O balanço privado apresenta melhora de 
aproximadamente 0,23 pontos percentuais do PIB. Isto indica que o processo expansionário foi capaz de gerar poupança para acomodar o choque de demanda. No que tange ao resultado do governo, observa-se um arrefecimento. Além disso, estima-se que o saldo externo decresça apenas 0,13 pontos percentuais após o choque. Isso ocorreu porque as importações aumentaram com o crescimento do PIB e da apreciação cambial.

A modesta expansão econômica é causada pelo aumento do consumo que cresce 1,65\%. A redistribuição da renda em favor dos trabalhadores (classe com reduzida propensão marginal para poupar) estimula o crescimento do consumo. Na presença de excesso de capacidade produtiva e para atender ao aumento do consumo, os capitalistas reagem aumentando a produção. Como antes, o aumento da produção e dos lucros leva ao aumento do investimento, ao crescimento da produtividade do trabalho (via função de Okun), dos empregos e dos salários. O salário real cresce 0,47\%, apresentando a mesma taxa de crescimento da produtividade do trabalho. Esses fatores, conjuntamente, contribuem para a expansão da demanda, do produto e da inflação. Arnim e Rada (2011) e Cuesta (1990) encontraram resultados similares para mudanças na distribuição funcional da renda para Egito e Colômbia, respectivamente.

Acessando os resultados dessa simulação para a calibragem (2), observa-se que o produto também segue uma trajetória expansionária. Embora com elasticidades de comércio exterior elevadas, os resultados sejammenos expressivos. Como verificado anteriormente, isto ocorre porque os vazamentos (leakages) do sistema são maiores para a calibragem (2).

Em linhas gerais, o resultadoda transferência de renda governamental na economia é positivo e independente da calibragem adotada. A expansão produtiva pautada nos ganhos de produtividade, aumento do investimento, e com aumento salarial parece benéfica para a economia. Políticas redistributivas, portanto, podem ter um impacto positivo no nível de atividade econômica. $\mathrm{O}$ conflito distributivo, gerado pelo aumento dos salários nominais, é insuficiente para abortar a expansão. Como consequência disso, o governo deve continuar concentrando seus esforços nessa política para fomentar a expansão produtiva. Quanto ao choque cambial, os resultados não são tão positivos. O impacto da desvalorização cambial na atividade doméstica é sensível a calibragem adotada.

\subsubsection{A PROPOSTA NEOESTRUTURALISTA}

A última coluna da Tabela 3 exibe os resultados de uma depreciação de $10 \%$ da taxa de câmbio juntamente com um aumento de $10 \%$ das transferências do governo para os trabalhadores. Conforme o cenário (1), o qual trata comércio exterior como uma proporção fixa do produto, o experimento gera resultados contracionários. Após o cho- 
que, o PIB real decresce 1,71\%, e um processo deflacionário emerge. O resultado privado e o balanço do governo também deterioram. Seguindo a redução da renda, o resultado externo deteriora 1,35 pontos percentuais do PIB.

O resultado negativo do setor externo, devido ao aumento das importações, causou a redução da demanda e dos preços, condicionando os efeitos negativos desse experimento. A depreciação cambial aumenta o custo das importações quando as importações são preço inelásticas. A contração da demanda provoca a redução do emprego, dos salários, e da produtividade, reduzindo os preços finais e o produto. As transferências de renda do governo aos trabalhadores são insuficientes para conter o movimento contracionário. Assim, a redução do saldo externo e dos preços condicionam os resultados negativos desse experimento.

Em contraste, a calibragem (2) apresenta resultados expansionários. Dois canais explicam o movimento de expansão do produto nessa simulação. Primeiro, a transferência de renda para os trabalhadores estimula o crescimento do consumo, agindo como um fator propulsor do investimento e do produto. Segundo, o acréscimo das exportações tem encadeamentos internos na economia, causando o crescimento do emprego, dos salários, e do consumo. Este último cresce o equivalente a 2,13\%. Esses dois canais de estímulo da produção - via exportações e consumo doméstico - atuam como mecanismos impulsionadores do crescimento da economia.

Em síntese, os resultados da calibragem (2) apontam para a superioridade da política que combina redistribuição de renda com desvalorização cambial. Essa política tem o potencial para promover uma expansão econômica no curto e médio prazo, gerando o aumento do emprego e dos salários. Contudo, esses resultados positivos são condicionados pelos valores das elasticidades do comércio exterior. Nesse sentido, o próximo subitem examina em que condições políticas cambiais e redistributivas seriam fomentadoras do aumento da atividade econômica.

\subsubsection{ANÁLISE DE SENSITIVIDADE}

A Figura 1 apresenta os resultados dos exercícios de sensitividade. Painéis (a) e (b) mostram, respectivamente, os resultados para uma desvalorização cambial de $10 \%$ e uma política econômica que combina desvalorizações cambiais (10\%) com o aumento da transferências de renda de $10 \%$. Cabe frisar que o efeito dos choques no produto dependem da condição de Marshal-Lerner, das estatísticas do ano base, e da flexibilidade dos preços. O eixo das abscissas ilustra o intervalo das elasticidades-preço da demanda das importações e exportações $(0 \leq \phi, \varphi \geq 1)$; o eixo das ordenadas demonstra a taxa de crescimento do PIB real. 
Figura 1 - Resultados da análise de sensitividade (\%)

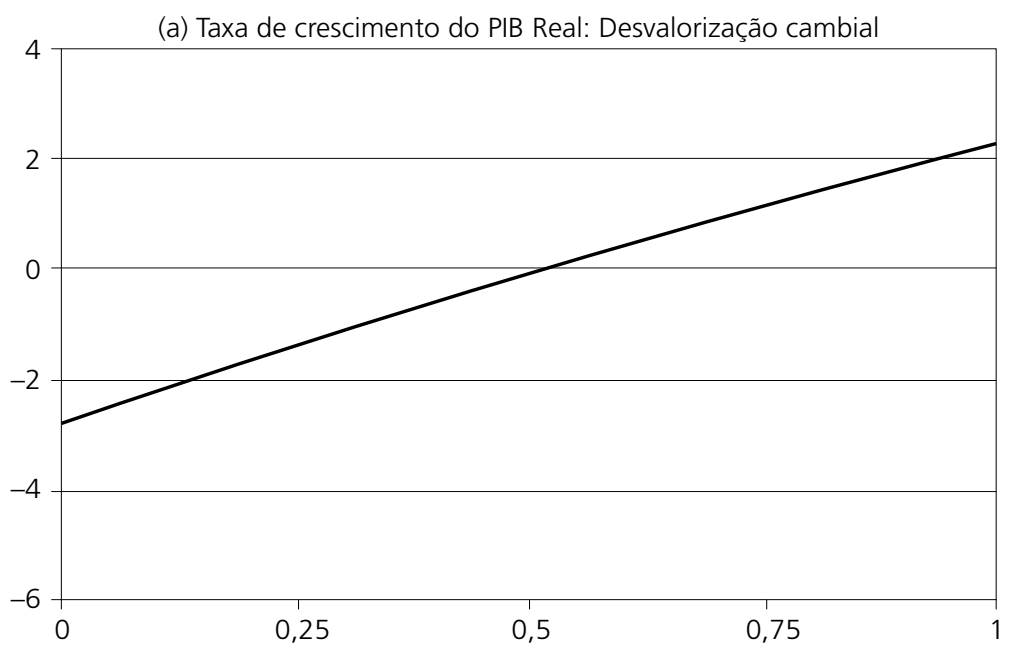

(b) Taxa de crescimento do PIB Real: Desvalorização e transferência

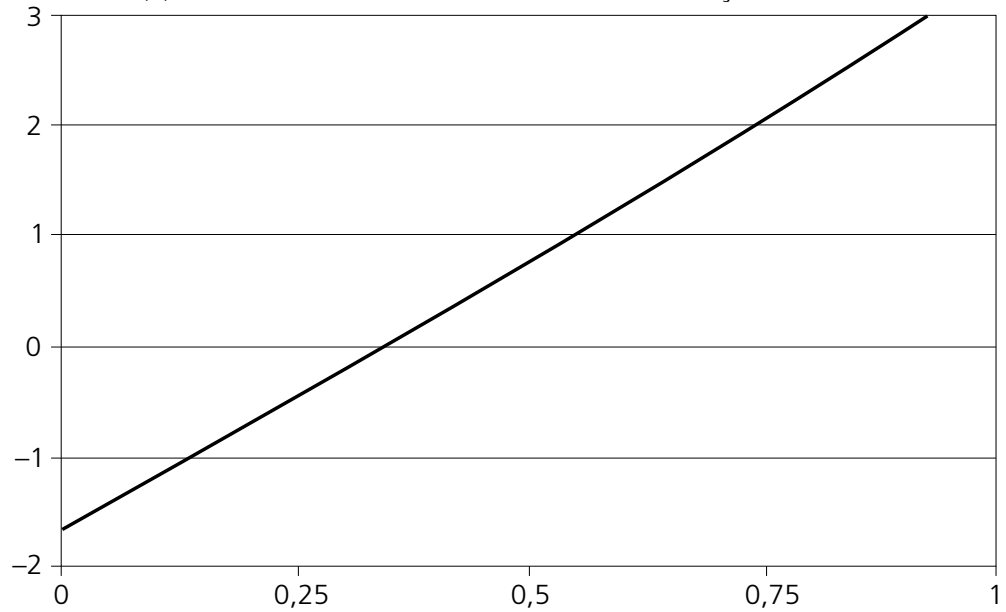

Nota: O eixo das ordenadas ilustra a taxa de crescimento do PIB real; o eixo das abscissas mostra as elasticidades preço da demanda de comércio exterior. As estimações foram realizadas usando-se o software Mathematica 8.0, o qual, em sua sintaxe de programação, não permite o uso de vírgulas entre números (apenas pontos). Painel (a) mostra o resultado de um aumento do investimento de $10 \%$ na taxa de crescimento do PIB real. Painel (b) mostra o resultado de uma desvalorização cambial de $10 \%$ combinada com o aumento da transferência de renda para os trabalhadores de $10 \%$. Fonte: Elaboração própria.

Os resultados dos painéis (a) e (b) indicam que, quanto maiores as elasticidades preço da demanda do comércio exterior, maior será o crescimento do PIB. Nesse sentido um ponto merece destaque. No painel (a) existe um limite a partir do qual uma depreciação cambial torna-se expansionária. Desvalorizações cambiais seriam contra- 
cionárias para elasticidades preço no intervalo de 0 a 0,54. A partir desse threshold $(0,54)$ depreciações tornam-se expansionárias. O processo expansionário ocorrerá se e somente se a economia brasileira apresentar elasticidades superiores a 0,54 . Isto adiciona incerteza quanto à eficácia da desvalorização cambial no curto e médio prazo tendo em vista que a economia brasileira possivelmente apresenta elasticidades preço inferiores a 0,54.

Examinando o painel (b), observamos que existe um limite a partir do qual depreciação cambial acompanhada de transferências produziria o aumento do nível da atividade econômica. Para elasticidades de comércio exterior no intervalo 0-0,28, a política econômica gera a contração da atividade econômica. Passando de 0,28, os resultados são expansionários. Isso indica a eficácia de desvalorizações cambiais sucedidas de políticas redistributivas para promover o aumento da atividade doméstica, minimizando o risco de haver uma contração produtiva. Assim, no contexto da economia brasileira de 2006, parece plausível supor que as elasticidades de exportação e importação sejam superiores a 0,28 , indicando a validade dessa política.

O presente estudo contribui ao mostrar que o debate wage-led/profit-led, no geral, e as desvalorizações cambiais, no particular, devem ser abordados no contexto de transferências de renda interinstitucionais. Nesse contexto, desvalorizações cambiais podem ser expansionárias mesmo no curto prazo se acompanhadas por transferências de renda do governo aos trabalhadores.

Em resumo, os resultados ratificaram a noção de que políticas de transferência de renda e cambiais podem gerar as condições iniciais para o progresso econômico. Quanto maior a elasticidade preço, maior será o estímulo para a expansão. Conforme os experimentos contrafactuais, sugere-se a execução com cautela de políticas cambiais e redistributivas para estimular a economia.

\section{CONCLUSÃO}

O fato de um cenário externo favorável seguido de políticas de infraestrutura e redistributivas ter causado o aumento da atividade econômica no período 2003-2008 não pode obscurecer o papel limitador do câmbio apreciado. Nesse sentido, o estudo apresentou um modelo para investigar se políticas de transferência de renda (em favor dos trabalhadores) juntamente com desvalorizações cambiais estimulam a economia. As estatística da economia brasileira para o ano de 2006 foram utilizadas para acessar os resultados empíricos do modelo macroeconômico.

Em linhas gerais, verifica-se que o aumento das transferências governamentais aos trabalhadores têm um impacto positivo na economia. Através do aumento da de- 
manda, a economia atinge um novo patamar, com maiores salários, investimento, produtividade e produção. No longo prazo, políticas redistributivas podem causar ganhos de saúde e acumulação de capital humano (Ranis e Stewart, 2000).

O choque de demanda (transferência de renda) estimula a atividade produtiva independentemente do cenário adotado. Esse resultado, juntamente com o maior espaço do governo para executar políticas heterodoxas, sugere que políticas distributivas deveriam ser intensificadas.

Em contraste, os resultados da desvalorização cambial na economia não são tão positivos, ensejando dúvida quanto ao efeito líquido dessa política. A desvalorização produzirá efeitos positivos na economia no curto e médio prazo se e somente se as elasticidades-preco de importações e exportações forem superiores a 0,54.

Quanto à política cambial e distributiva, pode-se afirmar que a ação conjunta dessas duas políticas reduz consideravelmente o risco de um resultado contracionário da ativividade produtiva. Essa política coordenada causará uma expansão se e somente se as elasticidades-preço de comércio exterior forem superiores a 0,28. Nesse sentido, sugere-se cautela na implementação de políticas cambiais, devendo a mesma ser combinada com políticas redistributivas a fim de evitar possíveis resultados contracionários.

Verificamos, portanto, que o aumento das transferências do governo aos trabalhadores e desvalorizações cambiais estimulam a produção doméstica. Os resultados dos experimentos contrafactuais revelam que essas políticas poderíam ter contribuído em um processo expansionário mais robusto da economia brasileira. O binômio câmbio desvalorizado-reditribuição deve ser empregado a fim de gerar o aumento da atividade econômica e a inclusão social.

\section{REFERÊNCIAS}

BADHURI, A.; MARGLIN, S. Unemployment and real wage: the economic basis for contesting political ideologies. Cambridge Journal of Economics, v. 14, n. 4, 1990.

BLECKER, R. A. “Kaleckian macro models for open economies”. In: DEPREZ, J.; HARVEY, J. T. (Eds.) Foundations of international economics: Post Keynesian perspectives. London: Routledge, 1999, p.116-149.

CARVALHO, V. R. S. A restrição externa e a perda de dinamismo da economia brasileira: investigando as relações entre estrutura produtiva e crescimento econômico. Dissertação de Mestrado, Faculdade de Economia, Universidade de São Paulo, São Paulo, 2005.

COMISSÃO ECONÔMICA PARA AMÉRICA LATINA E CARIBE (CEPAL). CEPALSTAT. [on-line]. 2012. Disponível em: <http://www. eclac. org/estadisticas >. Acesso em: 04 abr. 2013. 
CUESTA, J. L. L. "IS-FM macroeconomics: general equilibrium linkages of the food market in Colombia". In: TAYLOR, L. (Ed.) Social relevant policy analysis: structuralist computable general equilibrium models for the developing world. Cambridge: The MIT Press, 1990, p. 85-113.

DUTT, A. K. Stagnation, income distribution and monopoly power. Cambridge Journal of Economics, v. 8, n. 1, 1984.

FOLEY, D. Adam's fallacy: a guide to economic theology. Cambridge: Harvard University Press, 2008.

GRIJÓ, E.; BERNI, D. A metodologia completa para a estimativa de matrizes de insumo-produto. Teoria e Evidência Econômica, v. 14, n. 26, p. 9-42, mai. 2006.

GUILHOTO, J. J. M.; SESSO, U. Estimação da matriz insumo-produto a partir de dados preliminares das contas nacionais. Economia Aplicada, v. 9, n. 2, p. 277-299, abr./jun. 2005.

HOLANDA, M. C. Relações de longo prazo para as exportações e importações no Brasil. Texto para Discussão, Fortaleza, Universidade Federal do Ceará, n. 210, 1999.

INSTITUTO BRASILEIRO DE GEOGRAFIA E ESTATÍSTICA (IBGE). Tabela de Recursos e Usos. [on-line] Disponível em: <http: //www. ibge. gov. br/home/estatistica/economia/contasnacionais/2011>. Acesso em: 12 fev. 2013.

INTERNATIONAL LABOR ORGANIZATION (ILO). Studies on growth with equity. Brazil: An innovative income-led strategy. Geneva: International Labor Organization, International Institute for Labour Studies, 2011.

INSTITUTO DE PESQUISAS ECONÔMICAS APLICADAS (IPEA). Estatísticas sociais. [on-line] Disponível em: <http: //www. ipeadata. gov. br>. Acesso em: 03 mar. 2010.

KALECKI, M. Selected essays on the dynamics of the capitalist economy. Cambridge: CUP, 1971.

KRUGMAN, P.; TAYLOR, L. Contractionary effects of devaluation. Journal of International Economics,v. 8, n. 3, 1978.

LEONTIEF, W. Input-output economics. New York: Oxford University Press, 1986.

LEWIS, W. A. Economic development with unlimited supplies of labour. Manchester School, v. $28,1954$.

MCCOMBIE, J. S. L. Kaldor's law in retrospect. Journal of Post Keynesian Economics, v. 5, n. 3, 1983.

MORRONE, H. Three essays on distribution and economic expansion of a dual economy. Tese de Doutorado, Departamento de Economia,Universidade de Utah, Salt Lake City, 2012.

NAASTEPAD, C. W. M. Thecnology, demand and distribution: A cumulative growth model with an application to the Dutch productivity growth slowdown. Cambridge Journal of Economics, v. 30, n. 3, 2006.

OREIRO, J. L.; ARAUJO, E. Exchange rate misaligment, capital accumulation and income distribution: theory and evidence from the case of Brazil. Panoeconomicus, v. 3, special issue, 2013.

PALLEY, T. I. From Financial crisis to stagnation: the destruction of shared prosperity and the role of economics. Cambridge: Cambridge University Press, 2012. 
PREBISCH, R. Commercial policy in the underdeveloped countries. The American Economic Review, v. 49, n. 2, 1959.

STEINDL, J. Maturity and stagnation in American capitalism. New York: Monthly Review Press Classics, 1952.

THIRLWALL, A. P. A plain man's guide to Kaldor's growth laws. Journal of Post Keynesian Economics, v. 5, n. 3, 1983.

RADA, C. Stagnation or transformation of a dual economy through endogenous productivity growth. Cambridge Journal of Economics, v. 31, 2007.

RADA, C.; VON ARNIM, R. Structural transformation in China and India: A note on macroeconomic policies. Structural Change and Economic Dynamics, v. 23, n. 3, 2012.

RANIS, G.; STEWART, F. Strategies for success in human development. Journal of Human Development, v. 1, n. 1, 2000.

RUSKEEPAA, H. Mathematica Navigator: Mathematics, Statistics and Graphics. MA: Elsevier, 2009.

SANTOS, A. M. A.; SOUZA, E. A.; TEJADA, C. A. O.; JACINTO, P. A. Elasticidades preço e renda das exportações e importações: uma abordagem através de dados em painel para os estados do Brasil. In: Encontro Nacional da Associação Brasileira de Estudos Regionais e Urbanos, 7, 2009, São Paulo. Anais... São Paulo: ENABER, 2009.

TAYLOR, L.; BACHA, E. L.; E. A. CARDOSO; LYSY, F. J. Models of growth and distribution for Brazil. New York: Oxford University Press, 1980.

TAYLOR, L. Structuralist macroeconomics: applicable models for the Third World. New York: Basic Books, 1983.

TAYLOR, L. A stagnationist model of economic growth. Cambridge Journal of Economics, v. 9, 1985.

TAYLOR, L. Social Relevant policy analysis: structuralist computable general equilibrium models for the developing world. Cambridge: The MIT Press, 1990.

TAYLOR, L. Income distribution, inflation, and growth. Cambridge: The MIT Press, 1991.

UNITED NATIONS (UN). World economic situation and prospects 2010. New York: United Nations, 2010.

VON ARNIM, R. Wage policy in an open-economy Kalecki-Kaldor model: a aimulation study. Metroeconomica, v. 62, n. 2, 2010.

VON ARNIM, R.; RADA, C. Labour productivity and energy use in a three-sector model: an application to Egypt. Development and Change, v. 42, n. 6, 2011. 


\section{APÊNDICE: GLOSSÁRIO E EQUAÇÕES DO MODELO}

\section{VARIÁVEIS ENDÓGENAS}

$X_{t}$ : valor da produção (oferta);

$Y_{t}$ : produto interno bruto;

$v$ : parcela do valor adicionado na oferta total.

$Z_{t}$ : preço do valor adicionado;

$D Y$ : renda disponível, $\mathrm{i}=\mathrm{w}, \pi, \mathrm{f}$;

$C_{t}$ : consumo;

$S_{w}$ : poupança dos trabalhadores;

$S_{g}$ : poupança do governo;

$S_{\pi}$ : poupança externa;

$S_{f}$ poupança do capitalista;

$T$ : receita do governo;

$\xi$ : produtividade do trabalho;

$L_{t}$ : número de empregados;

$w_{t}$ : salário nominal;

$f$ : relação importações-produto;

$P_{t}:$ preço do produto final;

$E_{t}$ : exportações;

$I_{t}$ : investimento;

$r_{t}$ : taxa de lucro;

$\rho$ : taxa de câmbio real;

П: lucros.

\section{VARIÁVEIS EXÓGENAS}

$X_{f}$ demanda externa;

$L$ : força de trabalho;

$G_{t}$ : gastos públicos;

$\pi_{t}$ : parcela dos lucros no produto;

$y$ : taxa de mark-up;

$T R_{t}$ : transferência de renda das instituições para os trabalhadores;

$t t^{W}$ : impostos indiretos sobre o consumo dos trabalhadores;

$t t^{C}$ : imposto indireto incidente sobre os capitalistas; 
tte: impostos incidentes sobre as exportações;

$t t^{I}$ : impostos incidentes sobre o investimento;

$t t^{X}$ : impostos incidentes sobre a produção;

$t_{i}$ : taxa de impostos incidentes sobre capitalistas e trabalhadores, $i=c, w$;

$s_{i}$ : propensão marginal para poupar, $i=c, w$;

CapW: transferencia de renda dos capitalistas para os trabalhadores;

SubW: transferencia de renda do governo aos trabalhadores;

$R m W$ : remessa de renda do exterior aos trabalhadores;

$R m C$ : remessa monetária dos capitalistas para o exterior;

$e$ : taxa de câmbio nominal;

$P^{*}$ : preços do produto importado em moeda estrangeira;

$r m G$ : remessa de valores do governo ao exterior.

\section{PARÂMETROS}

$a_{t t}:$ coeficiente técnico de Leontief;

$\delta_{0}$ : parcela da mudança na produtividade não explicada por mudanças na demanda;

$\delta_{1}$ : elasticidade de Verdoorn;

$\delta_{2}$ : elasticidade do aumento nos custos sobre a produtividade;

$\phi_{1}$ : elasticidade preço das exportações;

$\varphi_{1}$ : elasticidade preço das importações;

$W_{1}$ : efeito do aumento do emprego nos salários nominais;

$W_{2}$ : efeito do aumento da produtividade na demanda dos trabalhadores por maiores salários;

$\chi_{0}:$ animal spirits;

$\chi_{1}$ : efeito da parcela salarial no nível de investimento;

$\chi_{2}$ : efeito do aumento do nível do produto no nível de investimento (acelerador).

\section{EQUAÇÕES DO MODELO}

1. Balanço Setorial

$$
a_{t t} X_{t}+C_{w t}+E_{t}+I_{t}+G_{t}-X_{t}=0
$$

2. Investimento

$$
I_{t}=\chi_{0}\left(\pi_{t} Y_{t}\right)^{x^{1}}\left(Y_{t}\right)^{x^{2}}
$$


3. Produtividade do Trabalho

$$
\xi=\delta_{0} Y_{t}^{\delta^{1}}\left(\frac{w_{t}}{Z_{t}}\right)^{\delta^{2}}
$$

4. Equações de Preços

$$
\begin{gathered}
Z_{t}=\frac{1}{(1-\pi)} w_{t} / \xi \\
P_{t}=\frac{v Z_{t}+f e}{1-a_{t t}}
\end{gathered}
$$

5. Mercado de Trabalho

$$
\begin{gathered}
L_{t}=\frac{Y_{t}}{\xi} \\
w_{t}=w_{0}\left(\frac{L_{t}}{L}\right)^{w_{1}} \xi^{w_{2}}
\end{gathered}
$$

6. Renda Disponível por Classes

$$
\begin{gathered}
D Y_{w}=\left(1-t_{w}\right)\left(\left(1-\pi_{t}\right) Z_{t} Y_{t}+T R\right) \\
D Y_{\pi}=\left(1-t_{c}\right)\left(\pi_{t} Z_{t} Y_{t}\right)-C a p W-r m C \\
D Y_{f}=e P^{*} F X_{t}+r m C+r m G-R m W-t t e-P t E t
\end{gathered}
$$

7. Equação da Demanda do Consumidor

$$
C_{t}=\frac{\left(1-t_{w}-s_{w}\right)\left(Z_{t} w_{t} L_{t}+C a p W+R m W+S u b W\right)}{P_{t}}
$$

8. Comércio Exterior

$$
\begin{aligned}
& E_{t}=\phi \rho^{\phi^{1}} X_{f} \\
& M_{t}=\varphi \rho^{\varphi^{1}} X_{t}
\end{aligned}
$$

9. Balanço Macroeconômico

$$
\begin{gathered}
S_{\pi}+S_{w}+S_{g}+S_{f}=P_{t} I_{t} \\
s_{\pi} \pi_{t} \frac{Z_{t}}{P_{t}} Y_{t}+s_{w} \frac{\left(1-\pi_{t}\right) Z_{t} Y_{t}}{P_{t}}+\frac{\left(T-P_{t} G_{t}-S u b W\right)}{P_{t}}+\frac{e P^{*} f X_{t}+r m C-t t e}{P_{t}}-E_{t}-\chi_{0}\left(\pi_{t} Y_{t}\right)^{\chi^{1}}\left(Y_{t}\right)^{\chi^{1}}=0
\end{gathered}
$$

